\title{
Screening Masses Close to the Chiral Phase Transition
}

\author{
G. Boyd ${ }^{a b *}$ \\ with S. Gupta ${ }^{b, c}$, F. Karsch ${ }^{a, b}$, E. Laermann ${ }^{a, *}$, \\ ${ }^{a}$ Fakultät für Physik, Universität Bielefeld, P.O. Box 100131, 33501 Bielefeld, Germany, \\ b HLRZ, c/o Forschungszentrum Jülich, 52425 Jülich, Germany, \\ c TIFR, Homi Bhabha Road, Bombay 400005, India
}

Evidence indicating that quarks are not confined inside mesons above the chiral transition is presented. Values are given for the effective four-fermion coupling between the quark and anti-quark above the chiral phase transition for both the pion and the rho meson.

\section{INTRODUCTION}

The possibility that the quarks may be confined in the pion above the chiral / deconfinement transition has received attention during the past few years [1,2]. The $\mathrm{MT}_{c}$ collaboration [3] has studied the chiral transition using four flavours of light staggered quarks on a lattice of size $16^{3} \times 8$. They found evidence for a first order chiral symmetry restoring phase transition. This appeared to be accompanied by a deconfinement transition, in that the rho had the screening mass expected for two free quarks. However, the pion screening mass was a factor of two lower, indicating that the pion could be a bound state.

The quark propagator also changes at the critical temperature, with the effective quark mass defined in the Landau gauge dropping by a factor of two at the phase transition [4]. This supported the idea that both the pion and the rho were probably deconfined above the chiral transition, but with different interactions between the quark and anti-quark in each meson channel.

The lowest Matsubara frequency of the quark on a lattice with temporal size eight, is large with respect to the effective mass in the high temperature phase. Hence it is difficult to extract a comparatively small binding energy.

If one uses periodic temporal boundary conditions for the valence quarks in the meson the low-

\footnotetext{
*Supported by Deutsche Forschungsgemeinschaft under grant Pe-340/3-1.
}

est Matsubara frequency becomes zero. So one may be able to gain additional insight into the nature of the mesons at high temperature. One expects to see no effect if the quarks are confined into the meson, but some effect if the quarks are not confined. (Note that the configurations are constructed using the usual anti-periodic temporal boundary conditions.)

Another complication lies in the point sources used for the meson operators. The meson correlator then has contributions from higher mass states, corresponding to the different internal quark momenta that can yield a fixed meson momentum. Hence one needs to follow the correlator to large distances. This then forces one to study the spatial correlators, and introduces the problem with the lowest Matsubara frequency alluded to above. So wall sources, which project each of the quarks in the meson onto half of the meson momentum, may enable one to extract a clean signal from the temporal correlator, yielding a measurement that is simpler to interpret.

\section{PERIODIC BOUNDARIES}

Correlators for the pion and rho mesons were constructed with both periodic and anti-periodic temporal boundary conditions for the valence quarks using the $\mathrm{MT}_{c}$ configurations, generated with anti-periodic temporal boundary conditions.

Below the phase transition, at $\beta=5.1$, neither the pion nor the rho propagator is affected by the 

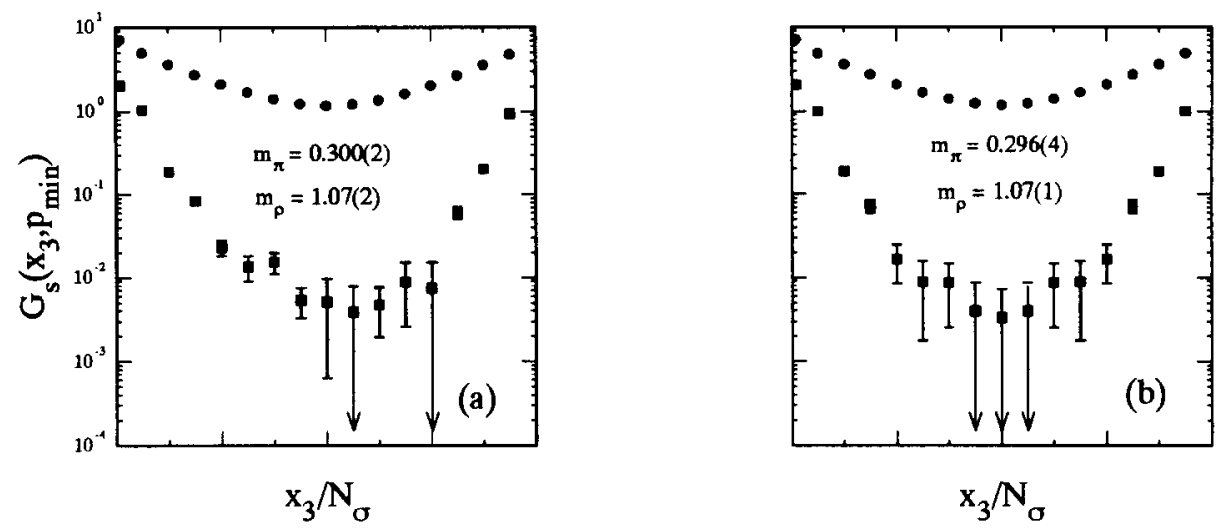

Figure 1. The spatial correlator in the PS (circles) and VT (squares) channels at $\beta=5.1$ with (a) anti-periodic and (b) periodic boundary conditions in the temporal direction. The masses shown were obtained from fits.
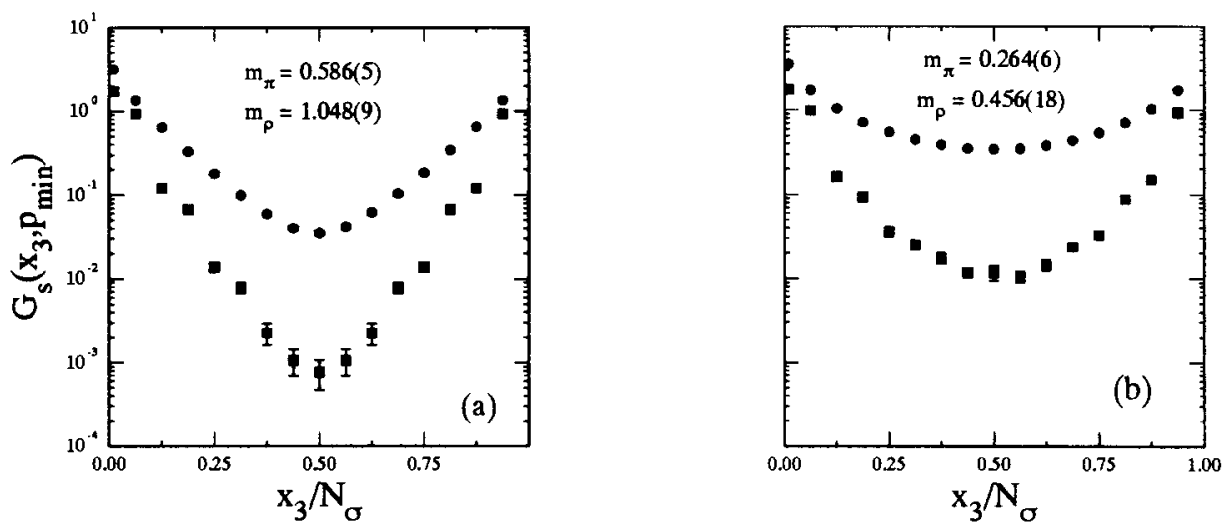

Figure 2. As for fig. 1, but at $\beta=5.3$.

change in boundary conditions (see fig. 1), as one expects if the quarks are confined into a meson.

The correlators in fig. 2 depict the correlators at about $1.8 T_{c}$. There is a large and qualitatively similar change in both the pion and the rho correlators. This indicates quite strongly that the quarks now contribute directly to the correlator, and are not confined inside the meson.

\section{WALL SOURCES}

One may calculate the correlators in both the temporal and spatial directions if one uses wall sources for the quarks. Since wall sources also fix the quark momenta, one should reach the asymptotic state sooner, as there is no need to damp out the contribution of the sum over internal quark momenta. This allows masses to be extracted from the temporal correlator as well.

These masses are depicted in fig. 3 along with twice the effective temporal quark mass [4]. The temporal correlator is not governed by the $2 \pi T$ associated with spatial propagation of quarks, and one is able to interpret the mass extracted from the correlator directly as an effective mass. One can see in fig. 3 that above the transition both the pion and the rho have masses near twice the effective quark mass.

The spatial correlation functions have also been examined, for both periodic and anti-periodic temporal boundary conditions, and also support the conclusion that above the chiral phase transition the quarks are not confined inside the 


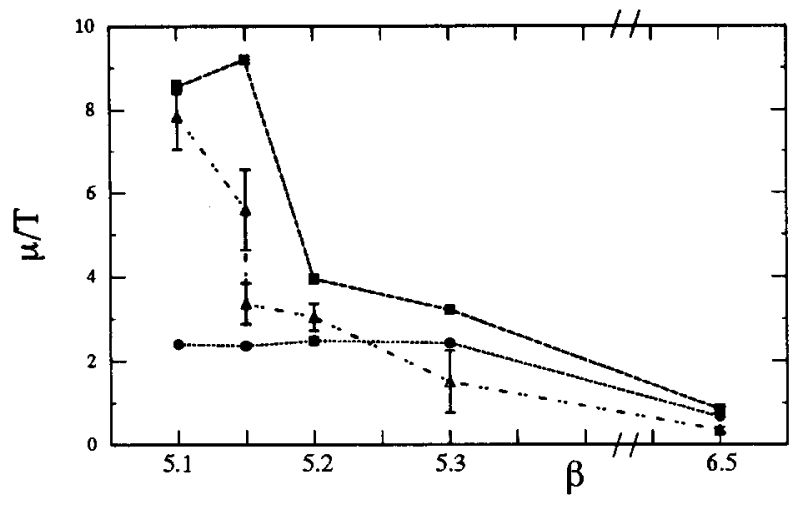

Figure 3. Local masses for the pion (circles), rho (squares) and twice the quark effective mass (triangles), extracted from the temporal correlator using wall sources.

mesons.

\section{FOUR FERMION COUPLINGS}

One may construct an effective four fermion coupling, eg. as in the Nambu-Jona-Lasinio model, from the correlator in momentum space, $\tilde{G}(p)$, as follows. Define the generalised susceptibility, $\chi$, equal to the correlator at zero fourmomentum and zero meson mass,

$\chi_{\pi, \rho}=\left.\tilde{G}_{\pi, \rho}(p=0)\right|_{m_{\pi, \rho} \rightarrow 0}$.

The susceptibility for non-interacting quarks in a given channel is denoted $\chi^{0}$.

One can then obtain the four fermion coupling $g_{\pi, \rho}$ from

$g T^{2}=\frac{N_{\tau}^{2}}{2}\left(\frac{1}{\chi^{0}}-\frac{1}{\chi}\right)$.

These are presented below, in table 1 . It is clear that, as expected from the correlation functions, the coupling in the pion channel is larger than in the rho channel.

\section{Conclusions}

All of the quantities examined indicate that above the chiral phase transition one has deconfined quarks, but with a considerable residual interaction. One does not reach the perturbative
Table 1

Four fermion couplings (up to an overall constant) in units of the temperature for the pion and rho channels above the phase transition.

\begin{tabular}{lll}
\hline$\beta$ & $g_{\pi} T^{2}$ & $g_{\rho} T^{2}$ \\
\hline $5.15(\mathrm{~S})$ & $16.27(11)$ & $3.77(21)$ \\
5.2 & $16.08(15)$ & $3.87(23)$ \\
5.3 & $15.23(27)$ & $3.77(31)$ \\
6.5 & $10(16)$ & $2.11(84)$ \\
\hline
\end{tabular}

regime until some way above the transition temperature.

The interaction between a quark - anti-quark pair carrying pion quantum numbers is about four times stronger than that in the rho channel. This is responsible for the difference between the pion and rho mass above the phase transition. Further details may be found in $[5,6]$.

\section{REFERENCES}

1. C. De Tar, Phys. Rev. D 32 (1985) 276.

2. C. Detar and J. B. Kogut, Phys. Rev. D 36 (1987) 2828.

3. K. Born ET AL., Phys. Rev. Lett. 67 (1991) 302.

4. G. Boyd, S. Gupta, and F. Karsch, Nucl. Phys. B 385 (1992) 481.

5. G. Boyd, S. Gupta, F. Karsch, E. LaerMANN, preprint HLRZ - 54/93.

6. S. Gup'ta, Phys. Lett. B 288 (1992) 171. 